\title{
Three Conjectures about the Number Systems and Their Conversion
}

\author{
Zhang Yanhong \\ Tianhe College of Guangdong Polytechnic Normal University, \\ Guangzhou, Guangdong 510540
}

\begin{abstract}
The conversion between Tetrad Notation and other notational systems has attracted people's attention; addition conjecture: if the maximum digit of base- $\mathrm{N}$ notation is $(\mathrm{N}-1)$, then $(\mathrm{N}-1)+(\mathrm{N}-1)$ is a binary digit, whose most significant digit is 1 and least significant digit is $(\mathrm{N}-2)$; multiplication conjecture: if the maximum digit of base- $\mathrm{N}$ notation is $(\mathrm{N}-1)$, then $(\mathrm{N}-1) *(\mathrm{~N}-1)$ is a binary digit, whose most significant digit is $(\mathrm{N}-2)$ and least significant digit is 1 ; simple conversion conjecture: given, the radix of two sorts of number system $N_{1}, N_{2}$ are $R_{1}, R_{2}$ respectively, $i$ is an integer greater than 2 or equals to 2 , so if $R_{1}=R_{2} i$, then some relatively simple relationship of conversion exists between these two number systems.
\end{abstract}

Keywords-Number System; Tetrad Notation; Addition Conjecture; Multiplication Conjecture; Simple Conversion Conjecture

\section{I.INTRODUCTION}

As we all know, all the biological NDAs consist of the four bases (nucleotides), namely adenine (A), thymine (T), guanine $(G)$, cytosine $(C)$. Naturally, it formed a tetrad composition, which is the same as the binary notation formed by the opening or closing motions of the semiconductor. Therefore, it is the most suitable for DNA computers to process information by using tetrad notation. With the tremendous development of DNA computers, tetrad notation attracts lots of attentions, and so does the conversion between the various number systems. The operation rules and mutual conversion of binary notation, octal notation, decimal notation, hexadecimal notation have been described maturely and accurately in all kinds of textbooks, so it is needless for me to talk about, and my main point is to study quaternary system and its conversion with other systems and put forward a conjecture of number system conversion.

By the way, what we usually say 1 hour is equal to 60 minutes, 1 minute is equal to 60 seconds is not a proper sexagesimal notation; 1 day equals to 24 hours, 1 year equals to 12 months is also not the proper base- 24 notation or base-12 notation. Obviously, all above is just a kind of unit conversion in our daily life, but base-N notation should have $\mathrm{N}$ digitals.

\section{GENERAL INSTRUCTION OF TETRAD NOTATION}

The only four tetrad notation digitals: $0,1,2,3$;

Tetrad notation's radix $=4$;

Tetrad notation's addition computes according to the rule "meet 4 advance 1 "

Tetrad notation's subtraction computes according to the rule "borrow 1 when 4 "

Tetrad Notation can be translated as Notation in English
Tetrad Notation figure $\mathrm{n}$ can be marked as (n) 4 or nT

A.The Addition and Multiplication of Tetrad Notation

Form1 Addition of Tetrad Notation

\begin{tabular}{|c|c|c|c|c|}
\hline+ & 0 & 1 & 2 & 3 \\
\hline 0 & 0 & 1 & 2 & 3 \\
\hline 1 & 1 & 2 & 3 & 10 \\
\hline 2 & 2 & 3 & 10 & 11 \\
\hline 3 & 3 & 10 & 11 & 12 \\
\hline
\end{tabular}

Form 2 Multiplication of Tetrad Notation

\begin{tabular}{|c|c|c|c|c|}
\hline$*$ & 0 & 1 & 2 & 3 \\
\hline 0 & 0 & 0 & 0 & 0 \\
\hline 1 & 0 & 1 & 2 & 3 \\
\hline 2 & 0 & 2 & 10 & 12 \\
\hline 3 & 0 & 3 & 12 & 21 \\
\hline
\end{tabular}

Form 3 Comparison Between Tetrad Notation and Binary Notation

\begin{tabular}{|l|l|}
\hline $\begin{array}{l}\text { Tetrad } \\
\text { Notation }\end{array}$ & $\begin{array}{l}\text { Binary } \\
\text { Notation }\end{array}$ \\
\hline 0 & 00 \\
\hline 1 & 01 \\
\hline 2 & 10 \\
\hline 3 & 11 \\
\hline
\end{tabular}

\section{B. Conjecture of Addition and Multiplication}

Conjecture 1: an addition conjecture on base-N notation (addition conjecture): if the maximum digit of base- $\mathrm{N}$ notation is $(\mathrm{N}-1)$, then $(\mathrm{N}-1)+(\mathrm{N}-1)$ is a binary digit, whose most significant digit is 1 and least significant digit is $(\mathrm{N}-2)$. For example, $3 \mathrm{~T}+3 \mathrm{~T}=12 \mathrm{~T}$ (Tetrad Notation), $7 \mathrm{O}+7 \mathrm{O}=16 \mathrm{O}$ (Octal Notation), $9 \mathrm{D}+9 \mathrm{D}=18 \mathrm{D}$ (Decimal Notation), $\mathrm{FH}+\mathrm{FH}=1 \mathrm{EH}$ (hexadecimal notation).

Conjecture 2: a multiplication conjecture on base- $\mathrm{N}$ notation (multiplication conjecture): if the maximum digit of base-N notation is $(\mathrm{N}-1)$, then $(\mathrm{N}-1) *(\mathrm{~N}-1)$ is a binary digit, whose most significant digit is $(\mathrm{N}-2)$ and least significant digit is 1 . For example, $3 \mathrm{~T}^{*} 3 \mathrm{~T}=21 \mathrm{~T}$ ( Tetrad Notation) , $7 \mathrm{O}^{*} 7 \mathrm{O}=61 \mathrm{O}$ (Octal Notation), $9 \mathrm{D} * 9 \mathrm{D}=8$ $1 \mathrm{D}$ (decimal notation), $\mathrm{FH}^{*} \mathrm{FH}=\mathrm{E} 1 \mathrm{H}$ ( hexadecimal notation).

Conclusion: the above two conjectures can't applied to binary notation 
Corollary: if the maximum digit of base- $\mathrm{N}$ notation is $(\mathrm{N}-1)$, then $(\mathrm{N}-1) *(\mathrm{~N}-1)+(\mathrm{N}-1)+(\mathrm{N}-1)+1=100$.

For example: $3 * 3+3+3+1=100($ tetrad notation)), $7 * 7+7+7+2=100$ (octal notation), $9 * 9+9+9+1=100($ decimal notation), $\quad \mathrm{F}^{*} \mathrm{~F}+\mathrm{F}+\mathrm{F}+1=100$ (hexadecimal notation).

Proof: according to the conjecturel and conjecture2, $(\mathrm{N}-1) *(\mathrm{~N}-1)+(\mathrm{N}-1)+(\mathrm{N}-1)=(\mathrm{N}-2) 1+1(\mathrm{~N}-$ $2)=(N-1)(N-1)$, obviously, $(N-1)(N-1)+1=100$ is a right proposition. Attention, the italic $(\mathrm{N}-2),(\mathrm{N}-1)$ is a digit of a base- $\mathrm{N}$ notation.

\section{III.CONVERSION BETWEEN TETRAD NOTATION AND OTHER NOTATIONS}

\section{A.Conversion between Tetrad Notation and Binary Notation}

a)The method of converting tetrad notation to binary notation

According to Form3, just use two binary digits to take the place of a tetrad digit.

[Example1]

(1) $3221 \mathrm{~T}=11101001 \mathrm{~B}$

(2) $0.213 \mathrm{~T}=0.100111 \mathrm{~B}$

(3) $3210.123 \mathrm{~T}=11100100.011011 \mathrm{~B}$

That is: $\rightarrow 11,2 \rightarrow 10,1 \rightarrow 01,0 \rightarrow 00$,decimal point will not be moved.

b)The method of converting binary notation to tetrad notation

For integers, from the right to the left, digits are divided into groups, each of which concludes two. If in the end of the left, only one digit is left, we can add a 0 , according to Form1, respectively, use the tetrad digits to take the place of them; but if for decimals, we can start from the right side of decimal point, from the left to the right, digits are divided into groups, each of which concludes two. If in the end of the right, only one digit is left, we can add a 0 , according to Form1, respectively, use the tetrad digits to take the place of them

[Example 2]

(1) $111101001 \mathrm{~B}=0111101001 \mathrm{~B}=13221 \mathrm{~T}(\mathrm{add}$ a 0 on the left side)

(2) $0.10101 \mathrm{~B}=0.101010 \mathrm{~B}=0.222 \mathrm{~T} （$ add a 0 on the right side)

(3) $11011000.10111=11011000.101110 \mathrm{~B}=3120.232 \mathrm{~T}$

That is: $11 \rightarrow 3,10 \rightarrow 2,01 \rightarrow 1,00 \rightarrow 0$, decimal point will not be moved.

\section{B. Conversion between Tetrad Notation and Hexadecimal} Notation

Form4 Comparison Between Tetrad Notation and Binary Notation

\begin{tabular}{|l|l|l|l|}
\hline $\begin{array}{l}\text { Tetrad } \\
\text { Notation }\end{array}$ & $\begin{array}{l}\text { Hexadecimal } \\
\text { Notation }\end{array}$ & $\begin{array}{l}\text { Tetrad } \\
\text { Notation }\end{array}$ & $\begin{array}{l}\text { Hexadecimal } \\
\text { Notation }\end{array}$ \\
\hline 00 & 0 & 20 & 8 \\
\hline 01 & 1 & 21 & 9 \\
\hline 02 & 2 & 22 & A \\
\hline 03 & 3 & 23 & B \\
\hline 10 & 4 & 30 & $\mathrm{C}$ \\
\hline
\end{tabular}

\begin{tabular}{|l|l|l|l|}
\hline 11 & 5 & 31 & D \\
\hline 12 & 6 & 32 & E \\
\hline 13 & 7 & 33 & F \\
\hline
\end{tabular}

a)The method of converting hexadecimal notation to tetrad notation

According to Form2, just use two binary digits to take the place of a hexadecimal digit.

[Example3]

(1) $32 \mathrm{E} 59 \mathrm{H}=0302321121 \mathrm{~T}$

(2) $0.5 \mathrm{D} 13 \mathrm{H}=0.11310103 \mathrm{~T}$

(3)6805.A87Q=12 $200011.222013 \mathrm{~T}$

That is: $\rightarrow 03,2 \rightarrow 02, \ldots, \mathrm{E} \rightarrow 32, \mathrm{D} \rightarrow 31$, decimal point will not be moved.

b)The method of converting tetrad notation to hexadecimal notation

For integers, from the right to the left, digits are divided into groups, each of which concludes two. If in the end of the left, only one digit is left, we can add a 0 , according to Form2, respectively, use the hexadecimal digits to take the place of them; but if for decimals, we can start from the right side of decimal point, from the left to the right, digits are divided into groups, each of which concludes two. If in the end of the right, only one digit is left, we can add a 0 , according to Form1, respectively, use the hexadecimal digits to take the place of them

[Example 4]

(1) $11300220 \mathrm{~T}=11300220 \mathrm{Q}=5 \mathrm{C} 28 \mathrm{H}$

(2) $0.0220133 \mathrm{~T}=0.022013 \quad 30 \mathrm{Q}=0.287 \mathrm{CH}$ (add a 0 on the right side )

(3) $302331121.122 \mathrm{~T}=\quad \begin{array}{llllll}03 & 02 & 33 & 11 & 21.12\end{array}$ $20 \mathrm{Q}=32 \mathrm{~F} 59.68 \mathrm{H}$

That is: $11 \rightarrow 5,30 \rightarrow \mathrm{C}, \ldots, 20 \rightarrow 8,33 \rightarrow \mathrm{F}$, decimal point will not be moved.

\section{Conversion between Tetrad Notation and Octal Notation}

Convert by using the binary notation as a bridge, that is tetrad notation $\longleftrightarrow \longrightarrow$ binary notation $\longleftrightarrow \rightarrow$ octal notation

D.Conversion between Tetrad Notation and Decimal Notation

a)The method of converting tetrad notation to decimal notation

Sum up according to the expansion of bit weights

b) The method of converting decimal notation to tetrad notation

For integers, keep the remainder after dividing the integer by 4 , while for decimals, keep the integral part of the result after dividing the decimal by 4 .

\section{IV.ANALYSIS OF THE DIFFICULTY IN CONVERSION OF} NUMBER SYSTEMS AND CONJECTURE OF THE REASONS

According to the above conversion of the number systems, it is the simplest of the mutual conversion between tetrad notation and binary notation, tetrad notation and hexadecimal notation, the conversion between tetrad notation and octal notation is a little more difficult, but the most difficult is relatively the most difficult. What are the reasons? 
Conjecture3 (simple conversion conjecture): given, the radix of two sorts of number system $\mathrm{N}_{1}, \mathrm{~N}_{2}$ are $\mathrm{R}_{1}, \mathrm{R}_{2}$ respectively, $i$ is an integer greater than 2 or equals to 2 , so if $\mathrm{R}_{1}=\mathrm{R}_{2}{ }^{1}$, then some relatively simple relationship of conversion exist between these two number systems.

Concrete method: firstly, we should make an equivalent comparison form of $\mathrm{N}_{1}, \mathrm{~N}_{2}$, in which the numbers of $N_{1}$ are single digits (total number is $R_{1}$, from 0 to $R_{1}-1$ ), but the numbers of $N_{2}$ are $i$ compositions of digit(totally $R_{1}$ sorts, from $00 \ldots 0$ to $\left(R_{2}-1\right)\left(R_{2}-1\right) \ldots$

$\left(R_{2}-1\right)$ ). If we convert $N_{1}$ to $N_{2}$, we just need to the $i$ compositions of digit of $\mathrm{N}_{2}$ to take the place of the equivalent digits of $\mathrm{N}_{1}$. On the contrary, if we convert $\mathrm{N}_{2}$ to $\mathrm{N}_{1}$, for integers, from the right to the left, digits are divided into groups, each of which concludes $i$. If in the end of the left, the left digit are not enough for $i$, we can add 0 , according to comparison form, respectively, use the $\mathrm{N}_{1}$ digits to take the place of them; but if for decimals, we can start from the right side of decimal point, from the left to the right, digits are divided into groups, each of which concludes $i$. If in the end of the right, the left digits are not enough for $\mathrm{i}$, we can add 0 , according to comparison form, respectively, use the $\mathrm{N}_{1}$ digits to take the place of them

The following shows the positive analysis of the above conjectures

\section{A.Tetrad Notation- Binary Notation}

$\mathrm{R}_{4}=\mathrm{R}_{2}{ }^{2}$, $\mathrm{i}=2$, the Form3 (see above), their mutual conversion follows the method provided by "the conjecture"

\section{B.Hexadecimal notation- Tetrad notation}

$\mathrm{R}_{16}=\mathrm{R}_{4}{ }^{2}, \mathrm{i}=2$, the Form4 (see above), their mutual conversion follows the method provided by "the conjecture"

\section{C.Octal Notation- Binary Notation}

$\mathrm{R}_{8}=\mathrm{R}_{2}{ }^{3}, \mathrm{i}=3$, the Form5 (see below), their mutual conversion follows the method provided by "the conjecture"

Form5 Comparison between Binary Notation and Octal Notation

\begin{tabular}{|l|l|}
\hline $\begin{array}{l}\text { Binary } \\
\text { Notation }\end{array}$ & $\begin{array}{l}\text { Octal } \\
\text { Notation }\end{array}$ \\
\hline 000 & 0 \\
\hline 001 & 1 \\
\hline 010 & 2 \\
\hline 011 & 3 \\
\hline 100 & 4 \\
\hline
\end{tabular}

\begin{tabular}{|l|l|}
\hline 101 & 5 \\
\hline 110 & 6 \\
\hline 111 & 7 \\
\hline
\end{tabular}

\section{D.Hexadecimal Notation- Binary Notation}

$\mathrm{R}_{16}=\mathrm{R}_{2}{ }^{4}, \mathrm{i}=4$, form6(see below), their mutual conversion follows the method provided by "the conjecture"

Form6 Comparison between Hexadecimal Notation and Binary Notation

\begin{tabular}{|l|l|l|l|}
\hline $\begin{array}{l}\text { Binary } \\
\text { Notation }\end{array}$ & $\begin{array}{l}\text { Hexadecimal } \\
\text { Notation }\end{array}$ & $\begin{array}{l}\text { Binary } \\
\text { Notation }\end{array}$ & $\begin{array}{l}\text { Hexadecimal } \\
\text { Notation }\end{array}$ \\
\hline 0000 & 0 & 1000 & 8 \\
\hline 0001 & 1 & 1001 & 9 \\
\hline 0010 & 2 & 1010 & A \\
\hline 0011 & 3 & 1011 & B \\
\hline 0100 & 4 & 1100 & C \\
\hline 0101 & 5 & 1101 & D \\
\hline 0110 & 6 & 1110 & E \\
\hline 0111 & 7 & 1111 & F \\
\hline
\end{tabular}

\section{V.STUDY OF NOVENARY NOTATION AND TERNARY} NOTATION

In the discussion of those theories, few people ever talked about novenary notation and ternary notation. Based on the three "conjectures" of this thesis, I think it is also suitable for novenary notation and ternary notation. According to the addition conjecture, 2Ter+2Ter=11Ter (ternary notation), $8 \mathrm{~J}+8 \mathrm{~J}=17 \mathrm{~J} \quad$ (novenary notation); according to multiplication conjecture, 2Ter*2Ter=11Ter (ternary notation), $8 \mathrm{~J} * 8 \mathrm{~J}=71 \mathrm{~J}$ (novenary notation). See form 7、8、9.

Form7 Ternary Notation Addition

\begin{tabular}{|c|c|c|c|}
\hline+ & 0 & 1 & 2 \\
\hline 0 & 0 & 1 & 2 \\
\hline 1 & 1 & 2 & 10 \\
\hline 2 & 2 & 10 & 11 \\
\hline
\end{tabular}

Form8 Ternary Notation Multiplication

\begin{tabular}{|l|l|l|l|}
\hline$*$ & 0 & 1 & 2 \\
\hline 0 & 0 & 0 & 0 \\
\hline 1 & 0 & 1 & 2 \\
\hline 2 & 0 & 2 & 11 \\
\hline
\end{tabular}

Form9 Novenary Notation Multiplication

\begin{tabular}{|l|l|l|l|l|l|l|l|}
\hline $1 * 1=1$ & $2 * 1=2$ & $3 * 1=3$ & $4 * 1=1$ & $5 * 1=5$ & $6 * 1=6$ & $7 * 1=7$ & $8 * 1=8$ \\
& $2 * 2=4$ & $3 * 2=6$ & $4 * 2=8$ & $5 * 2=11$ & $6 * 2=13$ & $7 * 2=15$ & $8 * 2=17$ \\
& & $3 * 3=10$ & $4 * 3=13$ & $5 * 3=16$ & $6 * 3=20$ & $7 * 3=23$ & $8 * 3=26$ \\
& & & $4 * 4=17$ & $5 * 4=22$ & $6 * 4=26$ & $7 * 4=31$ & $8 * 4=35$ \\
\hline
\end{tabular}




\begin{tabular}{|l|l|l|l|l|l|l|}
\hline & & & $5 * 5=27$ & $6 * 5=33$ & $7 * 5=38$ & $8 * 5=44$ \\
& & & & $6 * 6=40$ & $7 * 6=46$ & $8 * 6=53$ \\
& & & & & $7 * 7=54$ & $8 * 7=62$ \\
& & & & & & $8 * 8=71$ \\
\hline
\end{tabular}

Naturally, the conversion between novenary notation and ternary notation is very simple, because $\mathrm{R}_{9}=\mathrm{R}_{3}{ }^{2}, \mathrm{i}=2$, form10 (see below), their mutual conversion follows the method provided by "the conjecture"

Form10 Comparison between Novenary Notation and Ternary Notation

\begin{tabular}{|l|l|l|l|}
\hline $\begin{array}{l}\text { Ternary } \\
\text { Notation }\end{array}$ & $\begin{array}{l}\text { Novenary } \\
\text { Notation }\end{array}$ & $\begin{array}{l}\text { Ternary } \\
\text { Notation }\end{array}$ & $\begin{array}{l}\text { Novenary } \\
\text { Notation }\end{array}$ \\
\hline 00 & 0 & 12 & 5 \\
\hline 01 & 1 & 20 & 6 \\
\hline 02 & 2 & 21 & 7 \\
\hline 10 & 3 & 22 & 8 \\
\hline 11 & 4 & & \\
\hline
\end{tabular}

[Example5 ]

(1) $8728 \mathrm{~J}=22 \quad 21 \quad 02 \quad 22$ Ter ( $\mathrm{J}$ stands for novenary notation, $\mathrm{T}$ stands for ternary notation)

(2) $0.325 \mathrm{~J}=0.100212 \mathrm{Ter}$

(3) $22220.22201 \mathrm{Ter}=020020.222010 \mathrm{Ter}=286.863 \mathrm{~J}$

That is: $3 \longleftrightarrow \longrightarrow 10,2 \longleftrightarrow \longrightarrow 02, \ldots, 8 \longleftrightarrow \longrightarrow 22,6 \longleftarrow \longrightarrow 20$, decimal point will not be moved.

So we can conclude, binary notation-base-32 notation, ..., ternary notation-base-27 notation, ..., tetrad notation-base-64 notation, ... , quinary notation-base- 25 notation, ..., all can have very simple mutual conversion.

\section{REFERENCES}

[1] MUELLER T.J. "The Influence of Laminar Separation and Transition on Low Reynold Number Airfoil Hysteresis(Translation Journals style)", Journal of Aircraft,vol22,1985,pp.764-770

[2] Michael S.Selig, James J.Guglielmo, Andy P.Broeren, " Experiments on Airfoils at Low Reynolds Number(Translation Journals style) " ,AIAA-1996-62,34th Aerospace Science Meeting and Exhibit,NV,Jan1996,pp.15-18

[3] TianshuLiu,K.uykendoll,R.Rhew,S.Jones , Avian Wings[J]. AIAA 2004-2186

[4] http://lbx777.com/gjc/ho/ho01.htm

[5] HUA Xin,GU Rui,JIN Jing-fu,etal. Numerical Simulation And Aerodynamic Performance Comparison Between Seagull Aerofoil and NACA 4412 Aerofoil under Low-Reynolds[J]. Advances in Natural Science, 2010,3 (2) : 244-250. 